\title{
Ciudadanía y construcción democrática
}

\section{Citizenship and democratic construction}

\author{
José David Buelvas Bruno ${ }^{1}$ \\ Universidad de Córdoba, Montería, Colombia
}

Recibido el 30 de enero de 2017, aceptado el 24 de julio de 2017

Disponible en internet el 12 de agosto de 2017

\begin{abstract}
Resumen: resulta complejo en la época actual, definir el concepto de ciudadanía y el rol de ciudadano frente a multitud de facetas de tipo extrínseco e intrínseco que hacen en extremo cambiante cualquier modalidad asociativa y cualquier creación de entidades ciudadaníaciudadanas. Esto, cada vez más se evidencia por multiplicidad de factores que en nuestro medio colombiano y en el más circunscripto, córdoba en donde la persistencia en el tiempo de la relación ciudadano-ciudad, se ve marcada e incidida casi de manera permanente por factores tan fuertes como el político. Todos los derivados del conflicto armado y social en unas múltiples vertientes que interfieren con mensajes poderosos en la relación ciudad- ciudadano e inclusive en la misma relación entre ciudadanos. Corresponde pues, tareas encomiables a entorno dirigencial, componer un marco conceptual que muy principalmente provea solución a las fracturas de las situaciones todas tangencialmente han ocasionado en el tejido social de nuestro entorno y que impone franca dificultad para la correcta expresión de una fluida relación ciudadano con sus pares principalmente, pero también con el estamento político, social y ciudadano.
\end{abstract}

\section{Introducción}

¿Qué es participación ciudadana? Con esta pregunta se inicia este trabajo monográfico ahondando en las diversas formas de participación que aparecen en la dinámica política de América Latina la cual presenta actualmente una extraordinaria paradoja. Por un lado, la región puede mostrar con gran orgullo más de dos décadas de gobiernos democráticos. Por otro, enfrenta una creciente crisis social. Se mantienen profundas desigualdades, existen serios niveles de pobreza, el crecimiento económico ha sido insuficiente y ha aumentado la insatisfacción ciudadana con esas democracias -expresada en muchos lugares por un extendido descontento popular-, generando en algunos casos consecuencias desestabilizadoras (PNUD, 2004).

\footnotetext{
1. Correo electrónico: buelvasb@yahoo.es
} 
Las cuales diezman las posibilidades de articular una eficiente manera de ejercer la Ciudadanía desde sus dimensiones social y política. Pues es en estas dimensiones donde residen gran parte de las problemáticas que se atribuyen a esta forma de organización social denominada Democracia. En el caso colombiano se puede afirmar existen hoy por hoy retos que se presentan ante las imposibilidades de adjuntar una ciudadanía participativa a una dinámica cultural de orden democrático, puesto que hay una disyunción entre este ejercicio de ciudadanía y la realidad, siendo esta ultima la más difícil de abordar, debido a las formas tan precarias de entendimiento social que se configuran como eslabones representativos de lo que es la realidad, sin ser en verdad la realidad misma.

Todo esto justificado en la urgencia que existe de demarcar con rigor y criterios académicos, el quehacer de la vida social y la estructuración de la Democracia en la cultura política.

\section{APROXIMACIÓN CONCEPTUAL A LA CONSTRUCCIÓN DE UN SIGNIFICADO DE CIUDADANÍA}

Sin lugar a dudas resulta complejo definir en la época actual, considerada desde la teoría sociológica contemporánea, ¿cuál es el papel que cumple o debe cumplir la ciudadanía y el ciudadano en concreto dentro de un conjunto general de circunstancias que rodean el mundo y la vida práctica de muchas sociedades, las cuales son consideradas desde esta misma teoría, sociedades líquidas?

Lo cual connota que se asiste a una inversión del sentido de esta ciudadanía, en cuanto el concepto proviene y se autodefine desde la etapa sólida de la modernidad, donde son también múltiples los problemas, pero la resolución es buscada desde otras aristas menos globales y ceñidas estructuralmente a las dinámicas sociales, culturales, políticas y económicas de un territorio, de una misma espacialidad donde conviven personas que se agrupan con propósitos definidos en base al encuentro, a la comunidad, lo vinculante y todas aquellas formas de incidencia que son las que vienen a constituir lo que es ser ciudadano. 
Así entonces, la definición de un marco conceptual y práctico es lo que más va a interesar en la articulación de la ciudadanía con la referencia del ciudadano en el contexto sociopolítico y cultural de un país como Colombia y en específico dentro del marco de referencia local en Montería, como una sociedad con una tendencia poblacional de ciudad intermedia, pues sus procesos sociales devienen dentro de un marco legal que se inscribe en un modelo social emergente, pues es muy reciente su constitución dentro del estado constitucional que surgió en el año 1991, el cual hasta nuestros días ha estado transitando por diversas esferas, entre ellas la de participación social y ciudadana en el estado social de derecho.

Donde la fractura histórica a causa del conflicto social y armado ha dado configuraciones a tal relación (Ciudadano y Ciudadanía) en cuanto una conlleva implícita una idea que logra estatuir una figura política y de derechos (ciudadano), mientras la otra está directamente vinculada a la forma como una sociedad en la conjunción de sus partes logra sacar adelante los propósitos que movilizan a grandes porciones de la población, bajo diversos mecanismos.

Ya sean estos de participación o de exigibilidad en el plano de los derechos tanto como en el de las funciones que debe cumplir toda aquella persona que viva en un país democrático, cuyas instancias sean definidas por medio de la estructura de los poderes públicos y de organizaciones de base social y comunitaria como Juntas de Acción Comunal, Asociaciones Barriales y gremios de reconocimiento social y político vinculadas a las dinámicas de acción colectiva y del territorio. Las cuales por demás son reconocidas en el contenido de la constitución política colombiana como parte activa dentro del desarrollo de una sociedad como la colombiana con una diversidad cultural también reconocida en este mismo escenario constitucional.

Lo cual también se ubica en el plano de derechos, pero se sostiene en la representación, y se lleva adelante mediante la aplicación de los diversos mecanismos de participación y de 
representación, pues lo que se quiere lograr de las instituciones y de las personas, entorno a lo público es siempre un mayor compromiso con la sociedad y sus diversos problemas.

Es así como al hablar de ciudadanía, se tenga necesariamente que hablar de ciudadano, sin olvidar que una categoría implica la otra y sin caer en errores básicos que lleven a confundir la relación de preexistencia de roles que hacen que se distingan una de la otra, por lo cual se aborda en este sentido el concepto de ciudadanía desde este trabajo como un resultado que arroja la puesta en escena de un modelo de ciudadano que es el Colombiano, en el escenario del conflicto social y sus manifestaciones alrededor de la vida social.

Puesto que ejercitar la ciudadanía como esa respuesta referenciada a las problemáticas que surgen en la sociedad, es ejercitar la capacidad instalada que tiene un Estado y sus legítimas fuerzas internas que se denominan poderes públicos para resolver con dinamismos propios los problemas y sostener por la vía de la participación las soluciones posibles para estos problemas, sean de orden social o de cualquier otro.

Los cuales dependen en gran medida de la reacción que una sociedad en relación a sus miembros pueda brindar al conjunto de la población y esta a su vez pueda valorar como una solución óptima que da respuesta acertada a las condiciones que se buscan generar.

Por lo tanto, al hablar de ciudadanía, las perspectivas son bastante amplias y se estructuran en base a criterios que se inscriben en las lógicas de la sociedad donde se desarrolla.

Bajo esta premisa se puede pensar que la ciudadanía deja de ser un atributo que se logra por una función que cumplen cada una de las personas que integran el país, para convertirse en algo que es necesario conquistar, como valor intrínseco a la dinámica social, cultural y política de una nación en relación a un territorio.

Lo que conlleva a tratar de dirimir los obstáculos que la impiden y hacen de su trayectoria algo invisible, como sucede en muchos países latinoamericanos, donde el ejercicio 
ciudadano se haya aislado de las problemáticas transversales que afectan el diario acontecer.

Hasta formalizar un estado de cosas, que se legitima en base a la concepción de ciudadanía, la cual según el planteamiento del profesor Antanas Mockus se debe entender como: “Un mínimo de humanidad compartida. Cuando decimos que alguien es ciudadano, pensamos en aquel que respeta unos mínimos, que genera una confianza básica. Ser ciudadano es respetar los derechos de los demás.

El núcleo central para ser ciudadano es, entonces, pensar en el otro. Se basa en tener claro que siempre hay un otro, y tener presente no sólo al otro que está cerca y con quien sabemos que vamos a relacionarnos directamente, sino también considerar al otro más remoto, al ser humano aparentemente más lejano -al desconocido, por ejemplo, o a quien hará parte de las futuras generaciones.

Todos podemos reconocernos como compañeros de un camino bien largo. Asimismo, ser ciudadano implica que se está a favor de los procesos colectivos. Ciudadano es el que se asocia, se organiza con otros ciudadanos y emprende acciones colectivas en torno a objetivos y tareas de interés común"?2.

Lo cual en escenarios de conflicto como en Colombia, resurge con un sentido que se manifiesta fragmentado, debido a que los problemas que golpean la vida en sociedad, se hacen evidentes en la lógica imperante del ejercicio de la ciudadanía.

Esto anterior nos lleva a comprender que el desarrollo de la vida en sociedad y el desarrollo de las vías de acceso a la ciudadanía, así como la articulación del ciudadano a la

\footnotetext{
${ }^{2}$ Antanas, Mockus. Profesor de la Universidad Nacional de Colombia, Columna de Opinión de la Pagina Web del Ministerio de Educación Nacional de Colombia; En: ¿Por qué competencias ciudadanas en Colombia? Apuntes para ampliar el contexto de la discusión sobre estándares y pruebas, que en competencias ciudadanas ha empezado a construir y aplicar el Ministerio de Educación.
} 
construcción de vínculos duraderos con los cambios y situaciones emergentes que se suscitan en comunidad son el producto de las consistentes formas en que las personas se pueden ayudar mutuamente.

En función ya sea de modificar los códigos con los que funciona la sociedad o de ejercer una función individual que se convierta en un estilo frecuente de encuentro cultural, pues esta última es la impronta que va a llevar a las comunidades a hallarse de frente con las posibilidades de transformación y de revolución que se necesitan, sin las cuales es imposible ver materialmente realizado el proyecto de ciudadanía y de ciudadano que muchas personas necesitan para convivir.

En este sentido, cabe analizar con Bauman algo que resulta crucial para entender el rumbo que tienen las lógicas sociales que imperan en el mundo contemporáneo, y es el destino, como él mismo lo sostiene de la desigualdad social en tiempos de lo que denomina modernidad líquida.

Lo cual está implícito en la forma de construcción de acciones colectivas y en la manera en que se impulsan éstas a la consolidación de comunidades que sean capaces de atender sus problemáticas, por medio de mecanismos más eficientes y cercanos a las lógicas de la sociedad en la que se construyan dichas acciones.

Existen casos de comunidades que se consolidan pioneras de logros y avances sociales importantes como por ejemplo en Colombia la comunidad de paz de la zona del Urabá Antioqueño, donde la asociatividad y la capacidad de cohesión de los pobladores pudieron resolver las problemáticas circunscritas a los embates del conflicto armado, configurando un magistral modelo de paz y desarrollo desde la base social, sin hacer uso de la fuerza y tampoco de mecanismos violentos, sino por medio solo de la unificación de criterios de acción colectiva, que hicieron posible la manifestación pública de intereses que habían aguardado en el silencio inescrutable de las tensiones y del conflicto. 


\section{1 ¿CÓMO DEFINIR EL CONCEPTO DE CIUDADANÍA Y EL PAPEL CIUDADANO?}

Para lograr dar una respuesta a esta pregunta resulta crucial volver la mirada entonces de manera retrospectiva en la historia misma, para entender a qué enfoque o segmento del amplio espectro del concepto de ciudadanía se refiere concretamente a la dimensión social y las implicancias que este tiene en la articulación a las tareas y compromisos que el ciudadano adquiere.

Pues el concepto de ciudadanía dice el antropólogo Colombiano Julián Arturo que, más allá de ser un concepto occidental, es un constructo político, jurídico, y ético ligado ante todo al surgimiento de los regímenes democráticos (JULIAN ARTURO et al, 2003, p, 18).

En ese orden entonces, cabe mencionar desde esta perspectiva como también señala el profesor Julián Arturo que;

"En los griegos la ciudadanía hacía referencia a una ciudadanía localizada en la polis como centro de las decisiones políticas y de la vida pública. En este sentido, las ciudades griegas materializaban las polis que dieron origen a las primeras formas de democracia en la antigüedad. En ellas residir en la ciudad no convertía a las personas en ciudadanos. Los miembros de las polis que tenían derecho a tomar parte de la vida política pública constituían el grupo privilegiado de los ciudadanos. Eran excluidos los esclavos, los obreros (artesanos) que por su condición no podían participar en los asuntos públicos y los extranjeros, que podían tener otra clase de libertades de comercio y movilización, pero no políticas. La mujer tampoco tampoco era ciudadana. Este modelo de ciudadanía es, pues, de escala reducida y discriminatoria, pero de gran importancia como punto de partida en la formulación conceptual de la ciudadanía. En el mundo antiguo, griego y romano, no se era ciudadano de forma automática sólo por el hecho de ser hijo de padre ciudadano. Realmente, había que hacerse ciudadano, es decir ello implicaba un proceso. Para hacerse ciudadano se requería pertenecer a una comunidad familiar ciudadana y ser presentado, desde pequeño, por sus padres a la comunidad gentilicia o fratría. Posteriormente, cuando el joven alcanzaba la mayoría de edad (veinte años) podía ser presentado en la comunidad política, lo que implicaba gozar de la plenitud de los derechos políticos. Para ser ciudadano era preciso tomar 
parte en la administración de los asuntos públicos: participar de las asambleas que legislaban y gobernaban las polis y que administraban justicia” (Julian Arturo; p, 19).

Este episodio de la historia conceptual, nos remite a un tipo de concepto de ciudadanía que puede vincularse con las primeras etapas de desarrollo de la modernidad, donde lo que seguía primando era el honor y no el reconocimiento.

Como algo que permitiese la estratégica funcionalidad del escenario social y de las exigencias culturales de transformación, que toda sociedad exige a sus asociados, es decir a aquellas personas que no solo habitamos una porción de tierra y de experiencia territorial, sino además hacemos que existan las fuerzas necesarias para empujar el vehículo del espíritu cultural de un tiempo y la naturaleza social de una comunidad.

En este sentido las claves para ejercitar la ciudadanía y de darle un rol al ciudadano, está enmarcado en la idea de Libertad e igualdad como componentes que llevan, desde la época griega a fabricar la democracia y la ciudadanía de cara a la actividad pública.

Por lo cual también ellos desarrollaron un sistema social donde las desigualdades sociales existentes tenían en la igualdad política de los ciudadanos su contrapeso y se constituían en el criterio primordial de la convivencia ciudadana. (Julián Arturo; p, 19) desde ahí era posible entender los derechos políticos a los cuales toda la sociedad le apostaba, sin olvidar que había de por medio el compromiso ineludible de ayuda mutua, colocándose restricciones a la individualidad y primando la colectividad como expresión.

Más adelante, veremos que la Ciudadanía en la modernidad tomada desde la etapa de la Ilustración y las revoluciones políticas del siglo XVIII en particular con la francesa, y en este sentido en un contexto de retroceso de los regímenes feudal monárquico y surgimiento de la sociedad industrial capitalista. En esta perspectiva, a ciudadanía se la asocia con la modernidad y las ideas e instituciones democráticas, como lo fue en la antigua Grecia (Julián Arturo; p, 21). 
En esta medida, es con las revoluciones desde donde se materializan cambios sustanciales que van en el orden de la idea de Ciudadanía y de comunidad, los cuales son conceptos críticos que van a desatar los verdaderos síntomas sociales de esta época.

Pues con ellos se cambia también la perspectiva y la lógica de hacer revoluciones, con lo que se reorienta el uso de los conceptos. Con las revoluciones políticas del siglo XVIII por ejemplo se remplazan el término "súbdito" por el de "ciudadano" y recupera para el ciudadano su doble característica: la de las libertades y la de la igualdad, en otras palabras, los derechos civiles y políticos. (Julian Arturo; p, 21).

Pero el surgimiento del concepto de ciudadanía en términos reales, obedece a un contexto que va a ser el del surgimiento de los estados-naciones, de ahí que se comience hablando en la modernidad de ciudadanía como el hecho de pertenecer desde el nacimiento o residencia a un Estado territorial.

Este es un límite que va a presentarse al concepto y a la dinámica de la sociedad en cuanto coloca raíces en la lógica de los gobiernos que regulan y conceden la ciudadanía. Sin dejarle libertad real a los miembros de la sociedad para que ellos mismos luchen y adelanten dignamente sus propios derechos asociados, teniendo en cuenta las condiciones que constitucionalmente, pueden surgir de la vida practica entre los que gobiernan y los gobernados.

Porque la ciudadanía implica una triple dimensión que viene a ser, jurídica, política y ética, como marco referencial que jalona las diferentes posturas que en este sentido se va a colocar como travesaño de cualquier dinámica social y cultural. 


\subsection{REPRESENTACIONES Y PARTICIPACIÓN SOCIAL EN COMUNIDADES Y ORGANIZACIONES} SOCIALES

Otra de las cuestiones bastante críticas en la perspectiva de la ciudadanía, es la consolidación de organizaciones sociales con base en dinámicas problemáticas que siguen cada comunidad y cada organización en sus territorios, las cuales son variadas y mutan la mayoría de las veces de un lugar a otro de un país a otro, lo cual dificulta el abordaje de su operatividad, por lo cual solo se hará referencia a aquellas maneras en que las comunidades y organizaciones sociales se erigen dentro del sistema de estado transnacional, lo cual ha hecho que gran parte de lo que significaba consolidar comunidad y forjar organizaciones sociales cambiara.

Por lo cual ahora hay que comenzar a configurar un concepto más aproximado de lo que es el Estado, del cual parte en gran medida el concepto de comunidad y de organización social que se quiera vincular a X o Y dinámica de ciudadanía, por eso partimos de señalar que:

"La organización y el dinamismo del sistema mundial se basan en la intervención de un conjunto de actores con características, intereses y valores diversos, que aplican determinadas estrategias de acción para intentar alcanzar sus objetivos. En la perspectiva geopolítica tradicional, el Estado se entendía como protagonista no solo principal, sino casi exclusivo, de unas relaciones internacionales calificadas por ello como estadocentricas. Su consideración como entidades políticas con personalidad jurídica, soberanía reconocida y un territorio delimitado por fronteras, así como principal detentador del poder en sus diferentes manifestaciones, capaz de condicionar por ello la acción de los restantes actores, fue la razón para ese protagonismo. La diplomacia o la guerra eran los medios principales que reflejaban esas relaciones interestatales de colaboración, competencia o conflicto, dentro de un sistema internacional marcado por grandes diferencias respecto a la fuerza e influencia de unos y otros". (Gutiérrez del Valle; 2011, p,109)

A partir de aquí es de donde surge esa nueva concepción de lo que es el estado, siendo los agentes transformadores del mismo, los actores que intervienen en dicha transformación, como las personas que cumplen un rol, no solo como ciudadanos sino además como 
miembros de la sociedad civil que tiene capacidad de asociatividad y crea los lazos fuertes con las estructuras que componen el Estado.

En este sentido la apuesta por la construcción de comunidades y de organizaciones de acción colectiva, sigue siendo un reto por adelantar, pues en la práctica es posible encontrar que la vida en comunidad y el desarrollo de la acción colectiva, se ha fragmentado en algunos lugares del mundo, donde el miedo social campea, donde la falta de organización de sociedad civil no es unitaria.

Lo cual conduce a perfilar y entender que el Estado, ha cambiado su forma de interacción con la ciudadanía, lo que se traduce en una dinámica de fragilización de la actividad de incidencia social. Siendo esto lo primordial para poder comprender además que la política es hoy día otra cuestión poco operativa y carente de lugar en la vida social de las personas que integran las comunidades.

En este sentido, dice ZygmuntBauman, que:

"El problema contemporáneo más siniestro y penoso puede expresarse más penosamente por medio del término "Unsicherheit", la palabra alemana que fusiona otras tres en español: "incertidumbre", "inseguridad" y "desprotección". Lo curioso es que la naturaleza de este problema es también un poderosísimo impedimento para instrumentar remedios colectivos: las personas que se sienten inseguras, las personas preocupadas por lo que puede deparar el futuro y que temen por su seguridad, no son verdaderamente libres para enfrentar los riesgos que exige una acción colectiva”. (BAUMAN; 2007, $\mathrm{p}, 13)$

De ahí que la experiencia de lo comunitario y lo organizativo, como experiencias de ciudadanía, tiendan a estar desconectadas de la dimensión política, por una razón fundamental y es la que ha llevado a exponer que la política se hace de cara a intereses que parten desde la individualidad y no desde la colectividad.

Esto significa que asistimos a una especie de caos iatrogénico, como señala también Bauman para explicar que esas condiciones del mundo contemporáneo, han producido además enfermedades que se convierten en malestares sociales más complejos, (pobreza 
extrema, desigualdades irreparables, exclusión sistemática, discriminación colectiva, violaciones de derechos laborales, derechos humanos desprotegidos, entre otros temas).

Siendo estos los nuevos modos cómo funcionaran las lógicas comunitarias y las de acción colectiva, hasta que los problemas derivados de esta experiencia superan las expectativas de las sociedades y se proponen acciones colectivas y toda clase de maneras de contrarrestar el surgimiento de los mismos, pero de espaldas a la realidad asociativa de los miembros que integran dichas sociedades.

Llevando a que muchas sociedades se vean inmersas en graves situaciones como sucede hoy con la Unión Europea, que de sus crisis internas vienen surgiendo otros problemas que colocan a la comunidad en tareas urgentes como la organización de protestas sociales frente a las demandas surgidas de la crisis. Casos como el movimiento de los indignados, tanto en Europa y parte de América Latina, así como los movimientos obreros y de campesinos, que buscan soluciones a sus demandas sociales y políticas.

En este sentido hablar del surgimiento de comunidades fuertes y sólidas es difícil por una razón específica y es la forma cómo algunas sociedades tienden a olvidarse de sus problemas, o simplemente se esquematizan en buscar soluciones que muy pronto cambian en relación al problema que inicialmente se pretendía solucionar.

Eso es lo que a Bauman le llama la atención, pues para él existen dificultades que siempre van a girar alrededor de la forma líquida que tienen los problemas, así como las personas en su dimensión de pensamiento, pues también es costoso según su planteamiento, que existan personas que puedan consolidar fuerzas y unirse en relación a solucionar de fondo un problema o de reclamar ante las instancias respectivas sus propios derechos.

Esta es una característica propia de las sociedades y organizaciones sociales actualmente, pues es notorio que muchas veces sus proyectos se ven truncadas o por la imponencia de 
fuerzas institucionales superiores en cuanto al poder decisional, o en relación a la manera como se dan las negociaciones que se pactan entre las instancias implicadas.

Por último, es preciso analizar también, el tipo de ciudadano que requiere un tiempo con esta configuración y el tipo o modelo de ciudadano que se ha venido construyendo desde hace unas décadas. Ante lo cual cabe expresar que:

"la alienación en las formas de vida contemporánea reviste nuevas y reformuladas expresiones que alimentan y dan continuidad a aquellas propiciadas por el orden propiamente moderno. Es así como el sujeto postmoderno se presenta aún más individualizado e inmerso en lógicas de consumo y explotación cada vez más fuertes. La sociedad capitalista, en su fase actual de producción postfordista, crea e instaura pautas de comportamiento y sistemas de significación social que otorgan una determinada interpretación a la realidad y así, en un sentido amplio, consigue fijar la forma en que los sujetos se acercan y se desenvuelven en ella. La organización social que se produce desde las nuevas lógicas del capital y la ampliación de los canales tecnológicos, evidencian claramente lo que Habermas detecta en las sociedades contemporáneas como la ruptura de los lazos intersubjetivos y comunicacionales, que impiden la formulación de consensos, pero, así mismo, de agrupación social, con lo cual se asiste a la individuación total y, por ende, al desconocimiento y extrañamiento del otro" (Moreno, Laura, 2008: p, 217-218).

Esto anterior ha llevado a que la expectativa sobre el acontecer de la ciudadanía gire y lo haga en el más absoluto silencio, hasta causar el efecto social de anulación de la fuerza y la expresión social articulada y sincrónica.

Aumentándose los índices sociales de alienación, enajenación y otros factores que degradan y sumergen a las poblaciones en un océano de permanentes desconciertos frente a la visibilidad de las situaciones y los roles que se deberían cumplir de cara a estos. En esta medida es que Bauman indica existe una sociedad liquida, puesto que ahora es difícil predecir más que antes, como será el futuro de muchas personas.

Siendo una característica fundamental de este tipo de sociedad con sus ciudadanos abordo, el hecho de una desmesurada expansión del consumo y un exagerado control del sujeto humano que se hace cosificador que rompe la posibilidad de mantener una fluida comunicación entre los mismos. 
Lo cual va a significar que hay necesidad de recuperar la confianza entre los pares y aquellos que sean desconocidos, puesto que hay nuevas exigencias que marcan y delinean el lindero de actuación para los ciudadanos, por donde obligatoriamente vamos caminando y la percepción es poco notoria de que se hace esto.

Así llegamos entonces a una postulación de nueva ciudadanía que va a ser la de una ciudadanía activa para que se realice el ejercicio de la democracia, donde la posible dominación institucional es reducida y reemplazada por búsquedas permanentes de unidad social, de acciones colectivas, de cambios sustanciales en la condición misional de los gremios estratégicos y los cívicos de las sociedades.

Referencias bibliográficas

Alvarado, S. (12 de Noviembre de 2014). http://unimilitardspace.metabiblioteca.org/bitstream/10654/12832/1/La\%20participaci\%C3\%B3n\% 20democr\%C3\%A1tica\%20en\%20Colombia\%20presentaci\%C3\%B3n\%20final.pdf . Obtenido de www.unimilitar-dspace.metabiblioteca.org

Araujo Oñate, R. E. (2011). Retos de la Democracia y de la participaciòn ciudadana. Bogota: Universidad del Rosario.

Biscaretti Di Ruffia, p. (1982). Las Instituciones legislativas de la democracia directa. Madrid: Tecnos.

Bobbio, N. (2001). El futuro de la democracia. México DF: FCE.

Congreso de Colombia. (6 de julio de 2015). Ley 1757. Por la cual se dictan disposiciones en materia de promoción y protección del derecho a la participación democrática. Bogotá, Colombia, Colombia: Congreso.

Congreso de la Republica. (1994). Ley 134 de 1994. Bogotá: Congreso de la República.

Echeverri, C. (2010). La participación Ciudadana en Colombia. Estudios de Derecho, 26.

Pardo, C. (2011). Reflexiones sobre el carácter participativo de. En R. Araujo, Retos de la participación (pág. 224). Bogotá: Universidad del Rosario.

Pinilla, R. E. (2016). Constitución política de Colombia, Concordada. Medellín: Sanchez editores. 
PNUD. (2004). La Democracia en America Latina. Bogotá: ONU.

Restrepo, D. (1995). La participación social como construcción del interés público. Nómadas, 3-9.

Reyes, P. (2009). Cultura Política en Latinoamérica y Colombia. En O. M. Quintana, Estatuto Epistemológico de la cultura Política (págs. 278-343). Bogotá: UNAL.

Sartori, G. (1998). Teoria de la democracia. Madrid: Alianza.

Velásquez, F. E. (2003). ¿Qué ha pasado con la participación ciudadana en Colombia? Bogotá: Fundación Corona.

Zuleta, E. (1998). La participación democrática en Colombia. Revista Universidad de Antioquia, 8. 